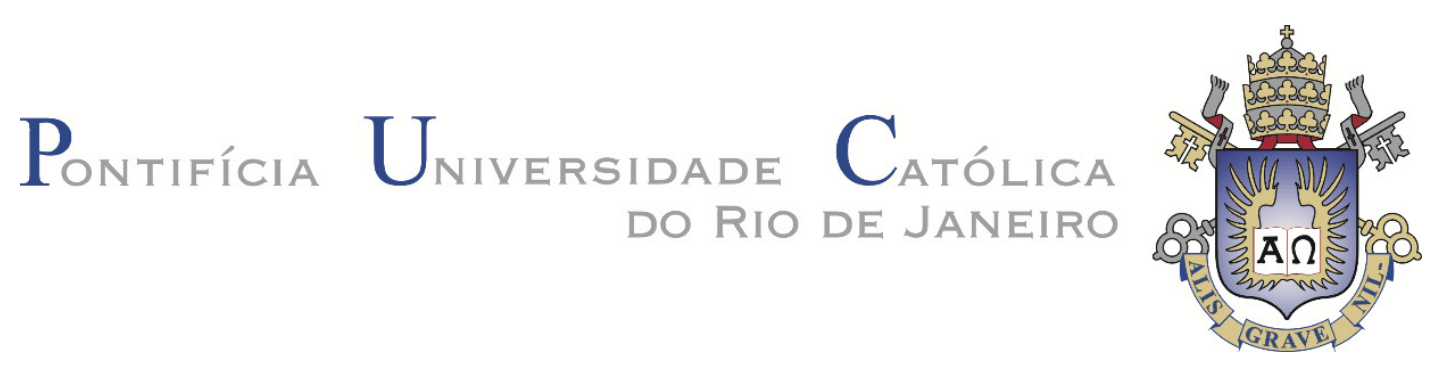

Joaquin Leonel Sanchez Salas

\title{
Modelo para Instabilidade e Vibrações de Placas Circulares
}

\section{Dissertação de Mestrado}

Dissertação apresentada como requisito parcial para obtenção do grau de Mestre pelo Programa de Pósgraduação em Engenharia Civil do Departamento de Engenharia Civil da PUC-Rio.

Orientador: Prof. Raul Rosas e Silva

Rio de Janeiro Junho de 2015 


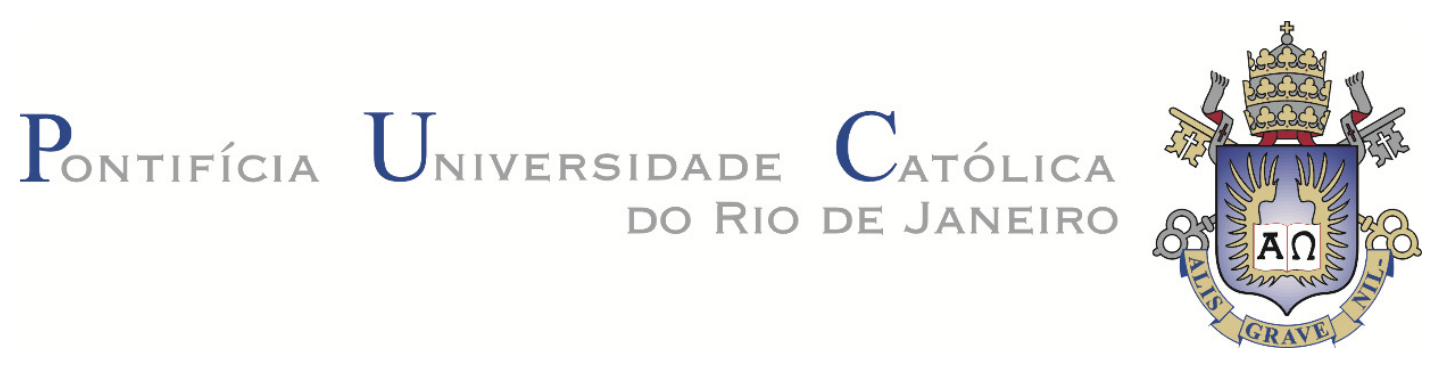

Joaquin Leonel Sanchez Salas

\section{Modelo para Instabilidade e Vibrações de Placas Circulares}

Dissertação apresentada como requisito parcial para obtenção do grau de Mestre pelo Programa de Pós-Graduação em Engenharia Civil do Departamento de Engenharia Civil do Centro Técnico Científico da PUC-Rio. Aprovada pela Comissão Examinadora abaixo assinada.

\section{Prof. Raul Rosas e Silva}

Orientador

Departamento de Engenharia Civil - PUC-Rio

Prof. Paulo Batista Gonçalves

Departamento de Engenharia Civil - PÚC-Rio

Prof. Carlos Alberto de Almeida

Departamento de Engenharia Mecânica- PUC-Rio

Prof. Glauco Jose de Oliveira Rodrigues Departamento de Engenharia Civil - PUC-Rio

Prof. Sebastiao Arthur Lopes de Andrade Departamento de Engenharia Civil - PUC-Rio

Prof. José Eugenio Leal

Coordenador Setorial do Centro Técnico Científico - PUC-Rio

Rio de Janeiro, 25 de junho de 2015 
Todos os direitos reservados. É proibida a reprodução total ou parcial do trabalho sem autorização do autor, da orientadora e da universidade.

\section{Joaquin Leonel Sanchez Salas}

Graduou-se em Engenharia Civil pela UPB (Universidade Privada Boliviana) em Cochabamba, Bolívia em maio de 2012. Ingressou no mestrado na Pontifícia Universidade Católica do Rio de Janeiro em março de 2013, desenvolvendo dissertação na linha de pesquisa de Instabilidade e Dinâmica das Estruturas.

Ficha Catalográfica

Sanchez Salas, Joaquin Leonel

Modelo para instabilidade e vibrações de placas circulares / Joaquin Leonel Sanchez Salas ; orientador: Raul Rosas e Silva. - 2012.

117 f. il. (color.) ; $30 \mathrm{~cm}$

Dissertação (mestrado)-Pontifícia Universidade Católica do Rio de Janeiro, Departamento de Engenharia Civil, 2015.

Inclui bibliografia

1. Engenharia civil - Teses. 2. Placa circulares e anulares. 3. Placa de espessura variável. 4. Carga conservativa e não conservativa. 5. Estabilidade de placas circulares. 6. Cargas seguidoras tangenciais não conservativas. 7. Método de Rayleigh-Ritz. I. Silva, Raul Rosas e. II. Pontifícia Universidade Católica do Rio de Janeiro. Departamento de Engenharia Civil. III. Título.

CDD: 624 
Aos meus pais e minhas irmãs. 


\section{Agradecimentos}

Para o meu orientador Raul Rosas e Silva, que me apoiou, me guiou e transmitiu muito conhecimento com todos os ensinamentos que foram passados em aulas cursadas no mestrado da PUC-Rio e pela orientação para o desenvolvimento desta dissertação.

Ao professor Glauco Jose de O. Rodrigues, pela contribuição do enfoque do tema da dissertação e pela disponibilidade em ajudar para o desenvolvimento deste.

Ao meu professor e amigo intimo Juan Ángel Ronda Vásquez, que foi o que despertou em mim a vontade de fazer o mestrado.

A minha família brasileira que souberam me acolher e me fazer parte da família. Obrigado, porque hoje posso falar que minha família cresceu até o Brasil e que ganhei pais e irmãos brasileiros.

A PUC-Rio por dar-me a oportunidade de poder cursar o mestrado nesta interinstituição muito reconhecida no brasil.

A todos os meus companheiros do mestrado, que me ajudaram a crescer como pessoa e a conhecer a diversidade de costumes e culturas do mundo (América Latina, Europa e África).

Obviamente, agradeço à minha família Boliviana, que sempre estiveram e estão no meu coração. Sem eles não teria feito nada do que consegui atualmente. Obrigado a minha mãe Magaly e meu pai Luis por me apoiarem neste sonho e obrigado as minhas irmãs Carola, Gabriela, Mariela, Cecilia (desde o céu) e Brenda por estarem presentes nos momentos que mais precisei.

A todos os professores e funcionários da PUC-Rio.

A CAPES por incentivar-me com uma bolsa de estudos, que me ajudou a realizar esta pesquisa. 


\section{Resumo}

Salas, Joaquin Leonel Sanchez; Silva, Raul Rosas e. Modelo para Instabilidade e Vibrações de placas Circulares. Rio de Janeiro, 2015. 117 p. Dissertação de Mestrado. Departamento de Engenharia Civil, Pontifícia Universidade Católica do Rio de Janeiro.

O presente trabalho mostra uma versão do método Rayleigh-Ritz com funções especializadas para a análise de placas circulares e anulares finas e espessas sujeitas a cargas fora do plano e em plano. As funções de aproximação para deslocamentos são polinómios em direção radial combinada com funções trigonométricas na direção circunferencial. Um recurso conveniente é o uso de funções nodais lineares, que permitem a fácil consideração de cargas nodais e condições de contorno (incluindo forças seguidoras), enriquecidos por polinômios de ordem superior, sem inclusão de nós adicionais. O modelo permite a variação da espessura e é aplicado em MAPLE18, possibilitando o cálculo de deslocamentos e tensões sob carregamento constante e de variação linear, as frequências de vibração, cargas de flambagem com alguns efeitos do nível de carga conservativa e não conservativa. Os exemplos mostram a eficácia desta abordagem na análise de tal estrutura e leva um novo enfoque a este problema clássico, que apresenta comparações interessantes e originais que descrevem o efeito de deformação de cisalhamento, no caso de vibrações o efeito das rotações inerciais e variação de espessura em placas circulares e anulares, incluindo deslocamentos, momentos e forças de cisalhamento, frequências de vibração, cargas de flambagem e uma análise de cargas seguidoras tangenciais não conservativas na estabilidade, utilizando o critério dinâmico é executada.

\section{Palavras-chave}

Placa circulares e anulares; placa de espessura variável; frequências naturais; carga conservativa e não conservativa; estabilidade de placas circulares; cargas seguidoras tangenciais não conservativas; método de Rayleigh -Ritz . 


\section{Abstract}

Salas, Joaquin Leonel Sanchez; Silva, Raul Rosas e (Advisor). A Model for Instability and Vibration of Circular Plates. Rio de Janeiro, 2015. 117 p. Msc. Dissertation. Departamento de Engenharia Civil, Pontifícia Universidade Católica do Rio de Janeiro.

The present work shows a version of the Rayleigh-Ritz method with specialized functions for the analysis of thin and thick circular and annular plates subjected to out-of-plane and in-plane loads. The approximation functions for displacements are polynomials in the radial direction combined with trigonometric functions in the circumferential direction. A convenient feature is the use of linear nodal functions, which allows for easy consideration of nodal loads and boundary conditions (including follower forces), enriched by higher order polynomials without inclusion of additional nodes. The model allows for thickness variation and was implemented in MAPLE18, enabling the calculation of displacements and stresses under constant and linearly varying load, frequencies of vibration, buckling loads with a few commands and the effect of the level of conservative and non-conservative on load the stability. The examples show the effectiveness of this approach in the analysis of such structures and bring new light to this classical problem, presenting interesting and novel comparisons illustrating the effect of shear deformation, in case of vibrations of the inertial rotations analysis and thickness variation in circular and annular plates, including displacements, moments and shear forces, vibration frequencies, buckling loads and a stability analysis of non-conservative tangential follower loads, using the dynamic criterion is performed.

\section{Keywords}

Circular and annular plate ; Variable plate thickness ; Natural frequencies; conservative and non-conservative load; Stability circular plates ; Non-conservative follower tangential loads ; Rayleigh -Ritz method. 


\section{Sumário}

$\begin{array}{lll}1 & \text { Introdução } & 19\end{array}$

1.1 Relevância e Justificativa da Pesquisa $\quad 19$

1.2 Objetivos 20

$\begin{array}{ll}1.3 \text { Estrutura da Dissertação } & 20\end{array}$

2 Desenvolvimento de Teorias de Placas 22

2.1 Considerações Iniciais 24

2.2 Placa Fina $\quad 26$

2.3 Placa Espessa $\quad 27$

2.4 Formulação do problema 28

3 Aplicação da Metodologia $\quad 37$

$\begin{array}{ll}3.1 \text { Placa fina } & 37\end{array}$

3.1.1 Campo de deslocamentos $\quad 37$

$\begin{array}{ll}\text { 3.1.2 Campo de deformações } & 37\end{array}$

3.1.3 Funções descritivas dos deslocamentos e graus de liberdade 38

3.1.4 Funções Nodais 39

3.1.5 Funções adicionais $\quad 40$

3.1.6 Energia de deformação da placa fina 40

3.1.7 Matriz de rigidez da placa fina 41

3.1.8 Vetor de forças 42

3.1.9 Matriz de massa $\quad 42$

3.1.10 Matriz geométrica $\quad 43$

3.2 Placa espessa $\quad 43$

3.2.1 Campo de deformações 43

3.2.2 Campo de deslocamentos 44 
3.2.3 Funções nodais $\quad 46$

3.2.4 Funções adicionais $\quad 46$

3.2.5 Graus de liberdade $\quad 47$

3.2.6 Relação tensão deformação 48

3.2.7 Energia de deformação da placa espessa 48

3.2.8 Matriz de rigidez da placa espessa 49

3.2.9 Vetor de forças $\quad 50$

3.2.10 Matriz de massa $\quad 50$

3.2.11 Matriz geométrica $\quad 50$

3.3 Matriz de rigidez dos apoios $\quad 51$

3.4 Frequências naturais $\quad 51$

3.5 Carga crítica 52

3.6 Matriz de carga seguidora $\quad 52$

3.7 Carga crítica dinâmica 52

3.8 Variação de espessura da placa 53

4 Exemplos $\quad 54$

4.1 Análise Estática $\quad 55$

4.1.1 Placa circular $\quad 55$

4.1.2 Placa circular anular $\quad 57$

4.2 Análise de Vibrações $\quad 67$

4.2.1 Placa Circular 67

4.2.2 Placa Circular Anular $\quad 67$

4.3 Análise de Instabilidade $\quad 73$

$\begin{array}{lll}\text { 4.3.1 Carga Crítica } & 73\end{array}$

4.4 Análise comparativa da carga crítica e frequências $\quad 74$

$\begin{array}{ll}4.5 \text { Carga crítica de Flutter (drapejamento) } & 78\end{array}$

5 Considerações finais $\quad 82$

5.1 Conclusões $\quad 82$

5.2 Sugestões para trabalhos futuros 83 
$\begin{array}{ll}\text { Anexo } & 87\end{array}$

A. Modelo Computacional para a obtenção de esforços e deslocamentos placa fina.

B. Modelo Computacional para a obtenção de esforços e deslocamentos placa espessa

C.Modelo Computacional para a obtenção de frequências da placa espessa 109

D. Modelo Computacional para a obtenção da carga crítica de flambagem da placa espessa.

E. Modelo Computacional para a obtenção da carga crítica de flambagem dinâmica da placa espessa. 


\section{Lista de Figuras}

Figura 2-1: Eixos Coordenados

Figura 2-2: (esquerda) Carregamento pontual, (direita) Tipo de carregamento axissimétrico, ambos os casos para uma placa circular.

Figura 2-3: (esquerda) Tipo de carregamento Axissimétrico, (direita) tipo de carregamento não axissimétrico, ambos os casos para uma placa circular anular.

Figura 2-4: Geometria de uma porção da placa com superfície media. 28

Figura 2-5: Características geométricas de uma seção da placa circular

Figura 2-6. Gráfica do estado de tensões em um elemento infinitesimal da placa.

Figura 2-7: Momentos e esforços cortantes do elemento infinitesimal.

Figura 2-8: Pressão axial uniformemente distribuída interna e externa da placa circular.

Figura 3-1: Graus de liberdade de a placa circular.

Figura 3-2. Relação de coordenadas normalizadas e coordenadas globais

Figura 4-1: Variação dos valores de carga crítica e frequência com relação à espessura da placa circular com bordo engastado.

Figura 4-2: Variação dos valores de carga crítica e frequência com relação à espessura da placa circular com bordo simplesmente apoiado.

Figura 4-3: Variação dos valores de carga crítica e frequência com relação à espessura da placa circular anular com o bordo interno engastado e o externo simplesmente apoiado.

Figura 4-4: Variação dos valores de carga crítica e frequência com relação à espessura da placa circular anular com o bordo interno e externo engastados. 
Figura 4-5: Coalescência das duas primeiras frequências da placa circular fina.

Figura 4-6: Coalescência das duas primeiras frequências da placa circular espessa.

Figura 4-7: Coalescência das duas primeiras frequências da placa fina circular anular.

Figura 4-8: Coalescência das duas primeiras frequências da placa espessa circular anular.

Figura 4-9: Variação da carga de flutter com relação da variação da espessura da placa circular.

Figura 4-10: Variação da carga de flutter com relação da variação da espessura da placa circular anular. 


\section{Lista de Tabelas}

Tabela 4-1: Comparação de resultados da placa circular com espessura constante e carregamento uniforme

Tabela 4-2: Comparação de momentos e deslocamentos da placa fina circular de espessura constante sujeita a uma carga pontual no centro da placa

Tabela 4-3: Comparação de deformação de uma placa fina de espessura variável ( $h 0 / h 1=1.50, \alpha=1.26, \beta=0.176, \beta 1=0,173$ e coeficiente de $v=0,25$ ) medidos no centro da placa.

Tabela 4-4: Comparação de esforços e deformação da placa circular de espessura constante sujeita a um carregamento não axissimétrico (em $r=6 m, \theta=0$ ).

Tabela 4-5: Comparação de esforços e deformações da placa anular de espessura constante sujeita a um carregamento uniforme.

Tabela 4-6: Comparação de deformação de uma placa anular de espessura variável $(a / b=3, h 0=0,2, h 1=0,6)$ sujeita a um carregamento distribuído, $v=0,3$

Tabela 4-7: Comparação das frequências da placa circular de espessura constante

Tabela 4-8: Comparação das frequências da placa circular anular de espessura constante

Tabela 4-9: Carga crítica de flambagem de placa circular de espessura constante

Tabela 4-10: Carga crítica de flambagem da placa circular anular de espessura constante

Tabela 4-11: Carga crítica de flambagem de aplaca circular anular de espessura constante 


\section{Lista de Quadros}

Quadro 4-1. Placa circular com bordo engastado sujeita a um carregamento pontual

Quadro 4-2. Placa circular com bordo simplesmente apoiado sujeita a um carregamento pontual

Quadro 4-3. Placa circular de espessura variável com bordo engastado sujeita a um carregamento distribuído

Quadro 4-4. Placa circular de espessura variável com bordo simplesmente apoiado sujeita a um carregamento pontual

Quadro 4-5. Placa circular de espessura constante engastado no bordo e sujeita a um carregamento não axissimétrico (linear)

Quadro 4-6. Placa circular de espessura constante simplesmente apoiado no bordo e sujeita a um carregamento não axissimétrico (linear)

Quadro 4-7. Placa circular anular de espessura constante com bordo interno engastado e externo livre sujeita a um carregamento constante

Quadro 4-8. Placa circular de espessura constante com bordo engastado

Quadro 4-9. Placa circular de espessura constante com bordo simplesmente apoiado

Quadro 4-10. Placa circular anular de espessura constante com bordo interno engastado e libre no bordo externo

Quadro 4-12. Placa circular anular de espessura constante com bordo interno e externo engastados 


\section{Lista de Abreviaturas}

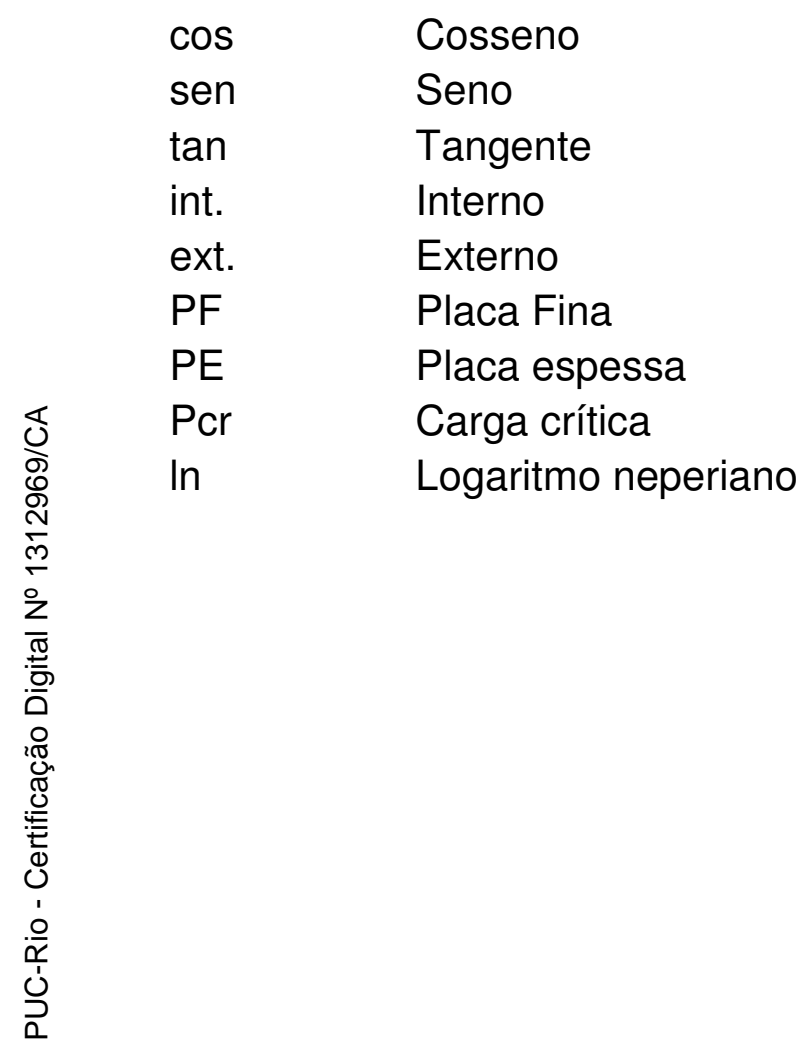




\section{Lista de Símbolos}

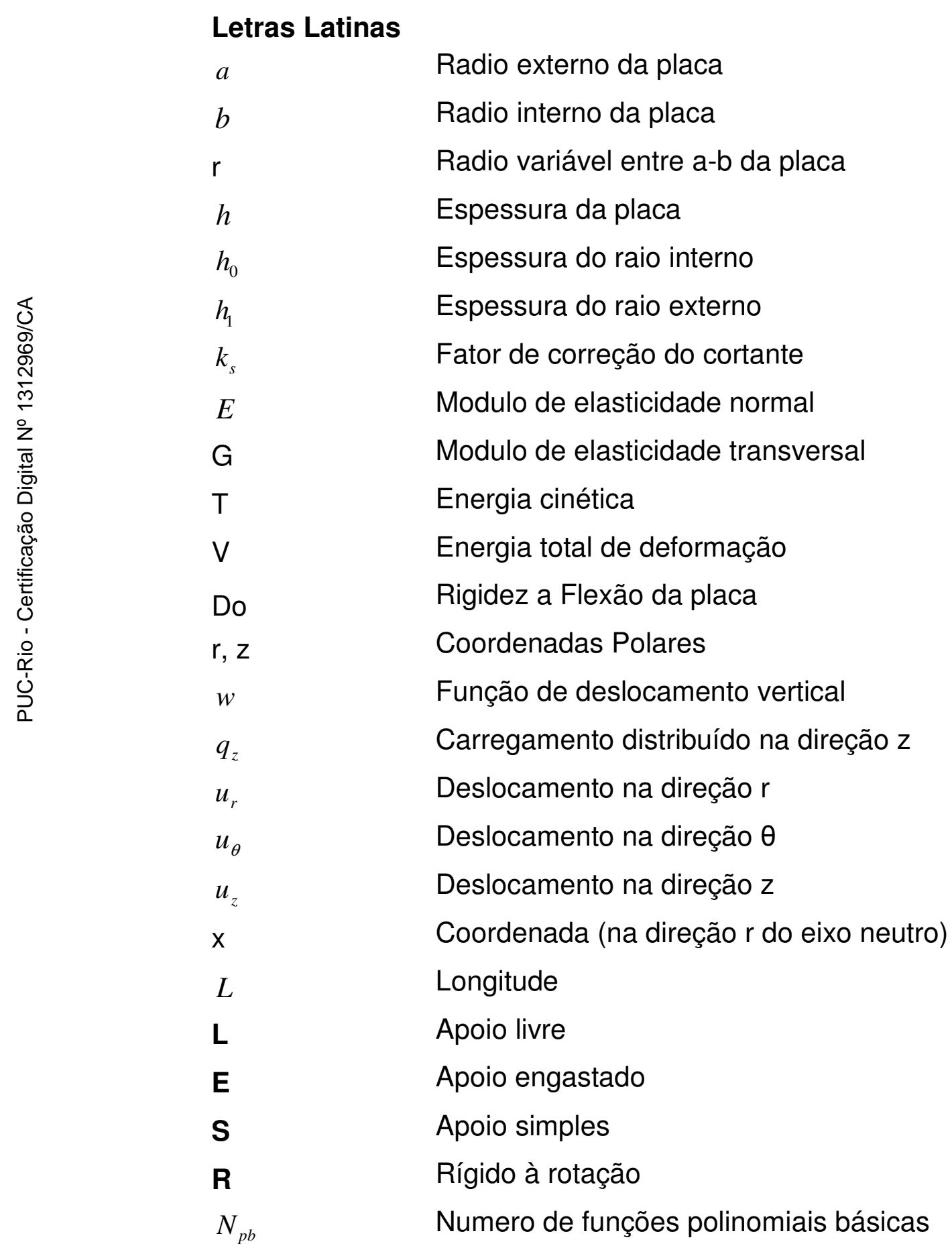




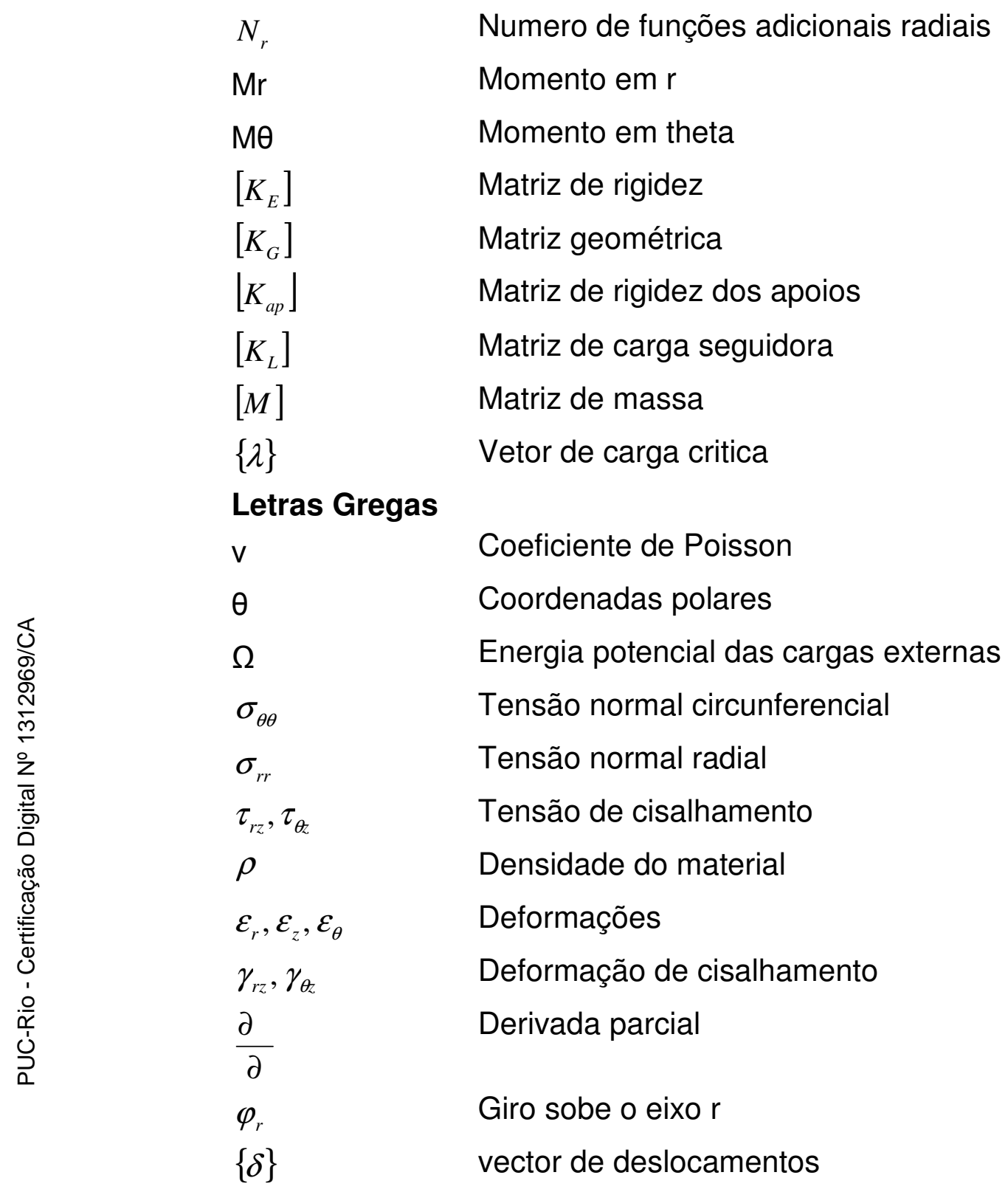


"Sin principio ni final solo energía variable con el tiempo"

Joaquin Leonel Sanchez Salas 